\title{
Polymer-based chemical nose systems for optical pattern recognition of gut microbiota
}

Shunsuke Tomita, ${ }^{*, 1,2,7}$ Hiroyuki Kusada, ${ }^{3,7}$ Naoshi Kojima, ${ }^{2}$ Sayaka Ishihara, ${ }^{2}$ Koyomi Miyazaki, ${ }^{4}$ Hideyuki Tamaki, ${ }^{3,5}$ and Ryoji Kurita ${ }^{* 1,2,6}$

${ }^{1}$ Health and Medical Research Institute, ${ }^{2}$ DBT-AIST International Laboratory for Advanced Biomedicine (DAILAB), DBT-AIST International Center for Translational \& Environmental Research (DAICENTER), ${ }^{3}$ Bioproduction Research Institute, ${ }^{4}$ Cellular and Molecular Biotechnology Research Institute, National Institute of Advanced Industrial Science and Technology, 1-1-1 Higashi, Tsukuba, Ibaraki 305-8566, Japan.

${ }^{5}$ JST ERATO Nomura Microbial Community Control Project, ${ }^{6}$ Faculty of Pure and Applied Sciences, University of Tsukuba, 1-1-1 Tennodai, Tsukuba, Ibaraki 305-8573, Japan.

${ }^{7}$ These authors contributed equally. 


\begin{abstract}
Understanding the status of gut microbiota has been recognized as crucial in health management and disease treatment. To meet the demands of medical and biological applications where rapid evaluation of gut microbiota in limited research environment is essential, we developed new sensing systems able to readout the overall characteristics of complex microbiota. Response patterns generated by a synthetic library of 12 charged block-copolymers with aggregation-induced emission units were analyzed with pattern recognition algorithms, allowing to identify the species/phyla of 16 axenic cultures of intestinal bacterial strains. More importantly, our method clearly classified artificial models of obesity-associated gut microbiota, and further succeeded in detecting sleep disorders in mice through comparative analysis of the normal/abnormal mouse gut microbiota. Our techniques can analyze complex bacterial samples far more quickly, simply and inexpensively than common metagenome-based methods, offering a powerful and complementary tool for gut microbiome analysis for practical use, e.g., in clinical settings.
\end{abstract}




\section{Introduction}

A number of diverse microorganisms [ $\sim 100$ trillion $\left(10^{14}\right)$ cells more than 1,000 species] inhabit the human gastrointestinal tract ${ }^{1,2}$. Recent studies have increasingly demonstrated that these microbial populations "gut microbiome" interacts with the host human, and thus closely related to our health and disease development ${ }^{3,4}$. A microbial imbalance, the so-called dysbiosis, has been observed in patients with a variety of diseases, including chronic sleep disruption, obesity, allergies, autism spectrum disorders, and cancers ${ }^{5-7}$. Therefore, understanding and controlling the gut microbiota are critical from the viewpoint of both health management and disease treatment.

Metagenomic analysis, the current standard method for gut microbiome analysis, involves the sequencing of $16 \mathrm{~S}$ ribosomal RNA (rRNA) gene amplicons or the entire genome of each bacteria in the microbiota. Although the metagenomics is a powerful tool, enabling to investigate whole community diversity and structure in the gut ecosystem, and genomic and metabolic capabilities of its constituents ${ }^{8,9}$, it does not necessarily meet the needs for medical and biological applications that demand rapid assessment of gut microbiome samples with limited equipment. This is because metagenomics requires highly expensive next-generation sequencer and high level of expertise for its use and data analysis in addition to significant labor and time. Besides, it still remains a challenge that the interpretations and insights obtained may differ from the actual state of the microbiota due to biases resulting from sample pretreatment, sequencing, and data analysis ${ }^{8,9}$.

In the process of exploring diagnostic and therapeutic approaches based on differences in gut microbiota between healthy/diseased individuals detected by metagenomic analysis, it has become clear that, despite the high complexity of the gut ecosystem, the gut microbiota exhibit

particular compositional patterns at the taxonomic rank level, e.g., genus-level enterotypes ${ }^{10-12}$ and 
phylum-level Firmicutes/Bacteroidetes $(F / B)$ ratio ${ }^{13-15}$, which correlate with the host conditions, including diseases and physical conditions. Inspired by these studies, we envisioned that such discrete compositional states can be recognized by sequencing-independent "chemical nose" sensing strategy. Chemical nose is an analytical concept that mimics the sensory mechanisms of animals, where arrays of molecular probes and pattern-recognition techniques are combined. By using a library of molecular probes that exhibit varying affinities for samples of interest, this strategy enables the generation of characteristic pattern information reflecting the entire samples through comprehensive interactions between molecular probes and the components of samples ${ }^{16,17}$. As with olfaction, the essence of this strategy is that the samples can be analyzed comparatively as long as the response patterns are generated, even if the compositions of the samples are unknown. Thus, it is suitable for determining whether a sample is normal or for classifying its state. Indeed, $\mathrm{we}^{18-20}$ and other researchers ${ }^{21,22}$ have demonstrated that this strategy is capable of identifying the states of complex biological samples, such as serum, cell lysates and secretions.

Here, we present the first chemical nose platform for recognizing gut microbiota. To challenge these largely unknown and obscure samples, we created a synthetic library of charged block-copolymers appended with aggregation-induced emission (AIE) fluorophores. Our chemical nose comprising up to 12 structurally diverse and environmentally sensitive polyethylene glycolblock-poly-L-lysine (PEG-b-PLL) derivatives can generate characteristic "fluorescence response patterns" through various interactions with the surfaces of intestinal bacteria (Figure 1A). The response patterns were subjected into pattern-recognition techniques, allowing to identify the gutderived bacterial strains at different taxonomic ranks (strains/species/phylum) and of mixed intestinal bacterial cultures as obesity models. Furthermore, this system could accurately distinguish the gut microbiota of healthy mice from those of insomniac mice. 
A

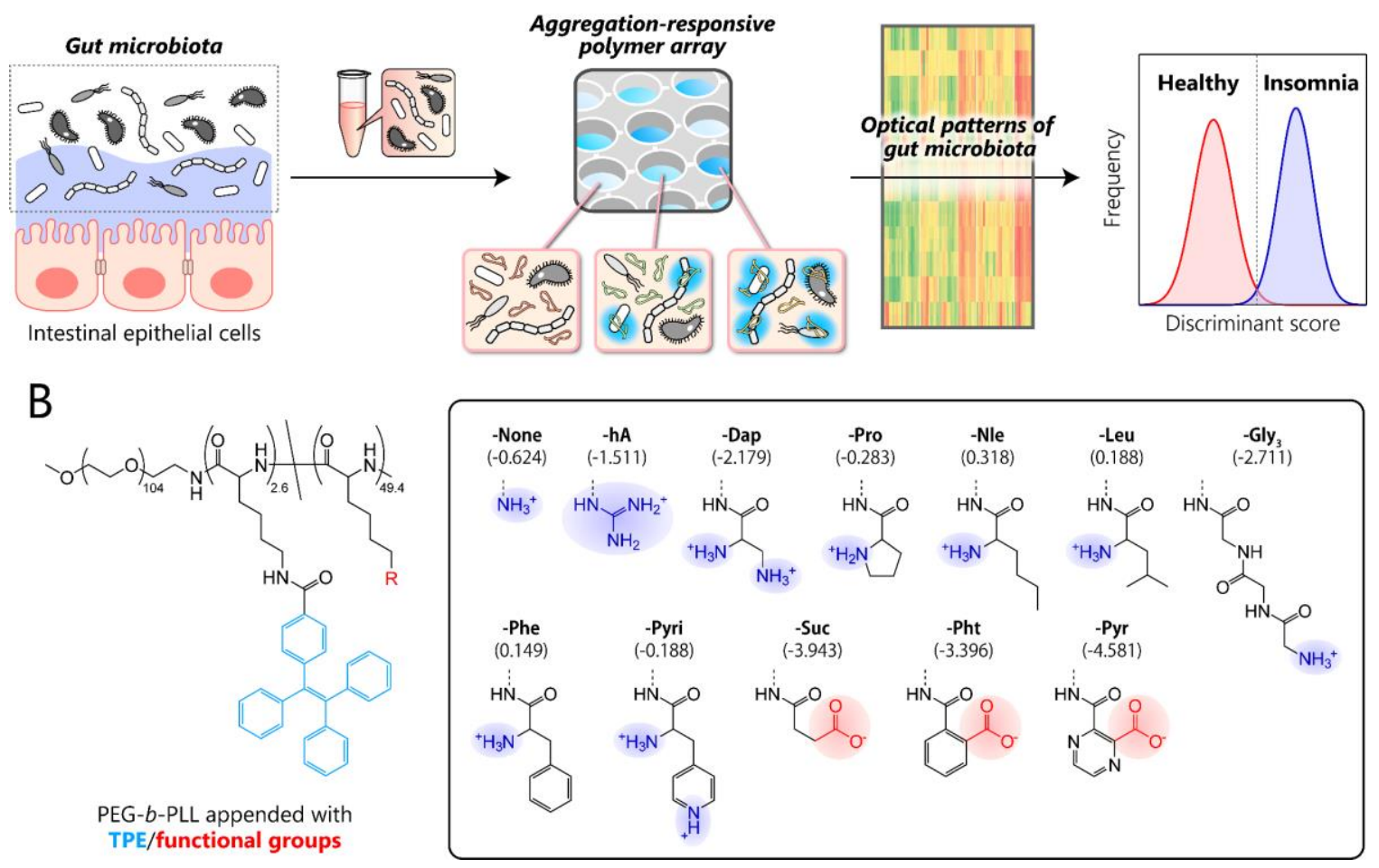

Figure 1. Workflow and synthetic library for optical pattern recognition of gut

microbiota. (A) The collected samples of mouse gut microbiota were applied to a chemical nose composed of aggregation-responsive polymers, generating fluorescence response patterns reflecting the characteristics of the whole gut microbiota, followed by statistical analysis with linear discriminant analysis. (B) The molecular structures of PEG- $b$-PLL appended with TPE fluorophores and various functional groups. $\log P$ values of the head groups are shown in parentheses.

\section{Results}

\section{Design and characterization of a polymer library}


A key in library design for the differentiation of highly complex gut microbiota (consisting of over 1,000 bacterial species) $)^{1,2}$ is to incorporate two classes of properties: (i) high chemical diversity capable of recognizing the surfaces of intestinal bacteria in a variety of manners and (ii) sharp turn-on emission characteristics capable of reproducibly transducing microenvironment of the bacterial surface into optical signals. Figure 1B illustrates a library of block-copolymers synthesized to meet the criteria. For the sensitive and selective extraction of bacterial characteristics, PEG- $b$-PLL was chosen as the scaffold material with high density of reaction sites that allow interaction in a multi-contact manner ${ }^{17,23}$. Amino groups of PEG- $b$-PLL were partially modified with an aggregation-induced emission (AIE) luminogen, tetraphenylethene (TPE) ${ }^{24}$. Upon binding to bacterial surfaces, PEG- $b$-PLL appended with TPE (-None) was expected to emit fluorescence through restricted intramolecular rotation of the central olefin stator of the TPE molecule. To provide high structural diversity, the remaining amino groups of -None were (i) guanidinized to enhance hydrogen-bonding ability (-hA), (ii) modified by amino acids with different aliphatic, aromatic, and additional amino groups (-Dap, -Pro, -Nle, -Leu, -Phe and Pyri) and a hydrophilic tripeptide (-Gly3), and (iii) modified by acid anhydrides for charge inversion from cationic to anionic (-Suc, -Pht and -Pyr). Since these modifications enable to cover a wide chemical space, we hypothesized that the array of these polymers can recognize complex features of bacterial surfaces consisting of lipopolysaccharides, lipids, peptidoglycans, and proteins $^{25}$, so that the surface information is transduced into a highly sensitive AIE due to the low background of the TPE fluorophore (For details of synthesis and characterization of the polymers, see Section 1 of the Supporting Information).

First, AIE response of TPE-appended PEG- $b$-PLL (-None) through its binding to bacteria was examined. While -None was nearly non-emissive in aqueous solution $(\mathrm{pH}=7.0)$, addition of 
Anaerostipes caccae (phylum Firmicutes; F.A., Table 1), an anaerobic and butyrate-producing intestinal bacterium, enhanced fluorescence emission up to nearly 40 -folds (Figure 2A). This response suggested that -None bound to or aggregated on the bacteria primarily through electrostatic interactions with the dense array of negatively charged teichoic acids present on the surface of the gram-positive $F . A .^{25}$, causing the restriction of intramolecular motion of TPE. The AIE feature of -None was also demonstrated by fluorescence microscopy, where blue emission from TPE was observed only from F.A. [the inset of Figures 2A, and S3 for Escherichia coli DH5 $\alpha$ (phylum Proteobacteria; P.E.1)], which is consistent with the microscopic observations of previous TPE derivatives for the pattern-recognition-based bacteria sensing ${ }^{26,27}$. The sharp turnon fluorescence capability of our TPE-appended polymer would lead to high experimental reproducibility due to high signal-to-background ratio, compared with our previously reported dansyl-fluorophore-modified polymers ${ }^{28}$, which is a stark advantage in constructing high-precision chemical noses.

Table 1. Gut-derived bacterial strains used for the initial testing.

\begin{tabular}{lllc}
\hline Phylum & Genus & Species & Abbr. \\
\hline Firmicutes & Anaerostipes & caccae & F.A. \\
Firmicutes & Blautia & hydrogenotrophica & F.B. \\
Firmicutes & Clostridium & citroniae & F.C. \\
Firmicutes & Eubacterium & fissicatena & F.E. \\
Firmicutes & Ruminococcus & gauvreauii & F.R. \\
Firmicutes & Lactococcus & lactis & F.L.1 \\
Firmicutes & Lactobacillus & helveticus & F.L.2 \\
Bacteroidetes & Bacteroides & dorei & B.B.1 \\
Bacteroidetes & Bacteroides & coprophilus & B.B.2
\end{tabular}




\begin{tabular}{llll} 
Bacteroidetes & Bacteroides & clarus & B.B.3 \\
Bacteroidetes & Bacteroides & oleiciplenus & B.B.4 \\
Actinobacteria & Bifidobacterium & longum & A.B.1 \\
Actinobacteria & Bifidobacterium & thermophilum & A.B.2 \\
Actinobacteria & Bifidobacterium & faecale & A.B.3 \\
Proteobacteria & Escherichia & coli $(\mathrm{DH} 5 \alpha)^{\mathrm{a}}$ & P.E.1 \\
Proteobacteria & Escherichia & coli $(\mathrm{JM} 109)^{\mathrm{a}}$ & P.E.2 \\
\hline
\end{tabular}

${ }^{\text {a }}$ Strain name.
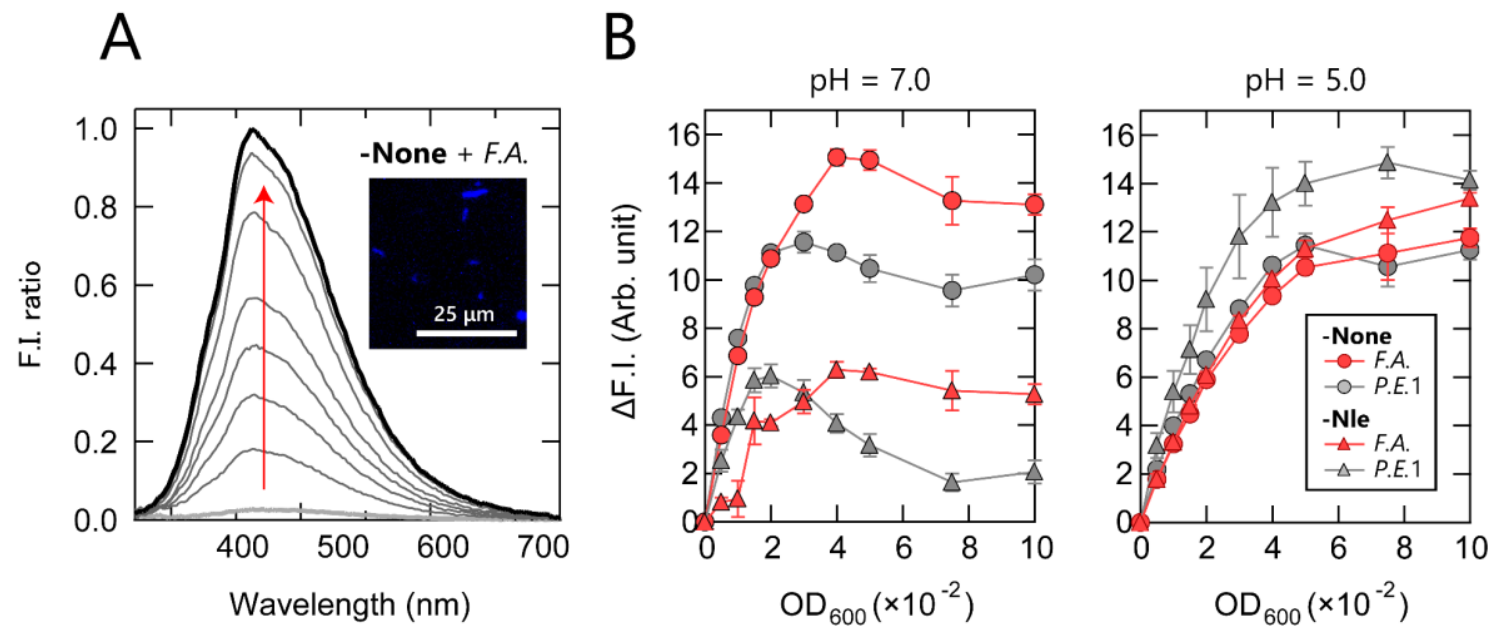

Figure 2. Characterization of representative polymers. (A) Fluorescence spectra of None $(150 \mathrm{nM})$ upon addition of $F . A .\left(\mathrm{OD}_{600}=0-0.05\right)$ in $20 \mathrm{mM}$ MOPS $(\mathrm{pH}=7.0) ; \lambda_{\mathrm{ex}}$ $=330 \mathrm{~nm}$. Inset: Fluorescence image of F.A. treated with -None. (B) Binding isotherms for -None and -Nle (150 nM) upon addition of F.A. and P.E.1 in $20 \mathrm{mM} \mathrm{MOPS} \mathrm{(pH} \mathrm{7.0)}$ and $20 \mathrm{mM}$ MES $(\mathrm{pH}=5.0)$ with $150 \mathrm{mM} \mathrm{NaCl} . \lambda_{\mathrm{ex}} / \lambda_{\mathrm{em}}=330 \mathrm{~nm} / 460 \mathrm{~nm}$. Values shown represent mean values $\pm 1 \mathrm{SE}(n=3)$.

We then investigated the relationship between the polymer structure and the response under physiological ionic strength (Figure 2B), where the relative contribution of other factors, such as 
hydrophobic interactions, to the signal was enhanced through electrostatic shielding. At $\mathrm{pH}=7.0$, -None experienced a similar increase in fluorescence intensity for F.A. and P.E.1 with optical density at $600 \mathrm{~nm}\left(\mathrm{OD}_{600}\right)<0.03$, but the maximum intensity was higher in $F . A$. than in P.E.1. A more complex behavior was observed for the hydrophobic -Nle. The responses to F.A. increased monotonically, while the signal began to decrease at $\mathrm{OD}_{600}>0.02$ in the case of P.E.1. In addition, the responses of charged polymers to bacteria varied markedly depending on the $\mathrm{pH}$ values; the responses of the polymers at $\mathrm{pH}=5.0$ became similar except for -Nle/P.E.1. These $\mathrm{pH}$ dependences appear to be attributed primarily to the protonation of the carboxyl groups on the bacterial surface and the amino groups of the polymer, especially for -Nle (Section 3 of the Supporting Information), by shifting the $\mathrm{pH}$ from 7.0 to 5.0. These complex responses were most likely due to differences in the chemical structure of the polymers, and this trend was expected to favor our polymer design for extracting features of bacterial strain and their mixed samples.

\section{Identification of gut-derived bacterial strains}

We tested whether our polymers could generate unique optical response patterns for gutderived bacterial strains (Table 1). The 16 bacterial strains chosen covered the predominant phyla in the gut microbiota, i.e., Actinobacteria, Bacteroidetes, Firmicutes and Proteobacteria, which constitute more than $97 \%$ of the human gut microbiome ${ }^{29}$. For the sensing procedure, each bacterial suspension was added to an array consisting of the polymers $(150 \mathrm{nM})$ in $20 \mathrm{mM}$ MOPS buffer $(\mathrm{pH}=7.0)$ or $20 \mathrm{mM}$ acetate buffer $(\mathrm{pH}=5.0)$ with $150 \mathrm{mM} \mathrm{NaCl}$ on a 96-well plate. The fluorescence responses from each bacterial strain/polymer combination were recorded using two different channels $\left[\lambda_{\mathrm{ex}} / \lambda_{\mathrm{em}}=330 \mathrm{~nm} / 480 \mathrm{~nm}(\mathrm{Ch} 1)\right.$ and $\left.360 \mathrm{~nm} / 530 \mathrm{~nm}(\mathrm{Ch} 2)\right]$, providing a dataset 
of 48-dimensional fluorescence patterns ( 12 polymers $\times 2 \mathrm{pHs} \times 2$ channels). A preliminary study has found that preprocessing of the data, usually required to improve the accuracy of chemical noses, such as background subtraction $\left(I-I_{0}\right)^{30}$, was not necessary (Figure S4), presumably due to the low background of TPE fluorescence. This is advantageous for the construction of a practical system as it saves labor and time.

The responses are summarized visually in the form of a heat map in Figure 3A. Applying the intestinal bacterial suspensions to our chemical nose obviously led to the generation of a variety of fluorescence response patterns. The discriminative information was then statistically evaluated using linear discriminant analysis (LDA), a supervised pattern recognition algorithm that provides a graphical output describing the similarity and the classification ability of the data ${ }^{16}$. The linear discriminant score plot (Figures 3B and S5), where each point represents the fluorescence response pattern of a single analyte in the chemical nose, confirmed well-separated clusters, indicating statistically significant differences between the patterns of the 16 bacterial strains examined. The discrimination accuracy was quantitatively evaluated by two different tests [a leave-one-out crossvalidation test (the so-called jackknife test) and a holdout test], and the $100 \%$ accuracy for the identification of the bacterial strains was successfully afforded in both tests. Furthermore, a minimal system with sufficient accuracy could be constructed using the selected two polymers (Section 4 of the Supporting Information). 

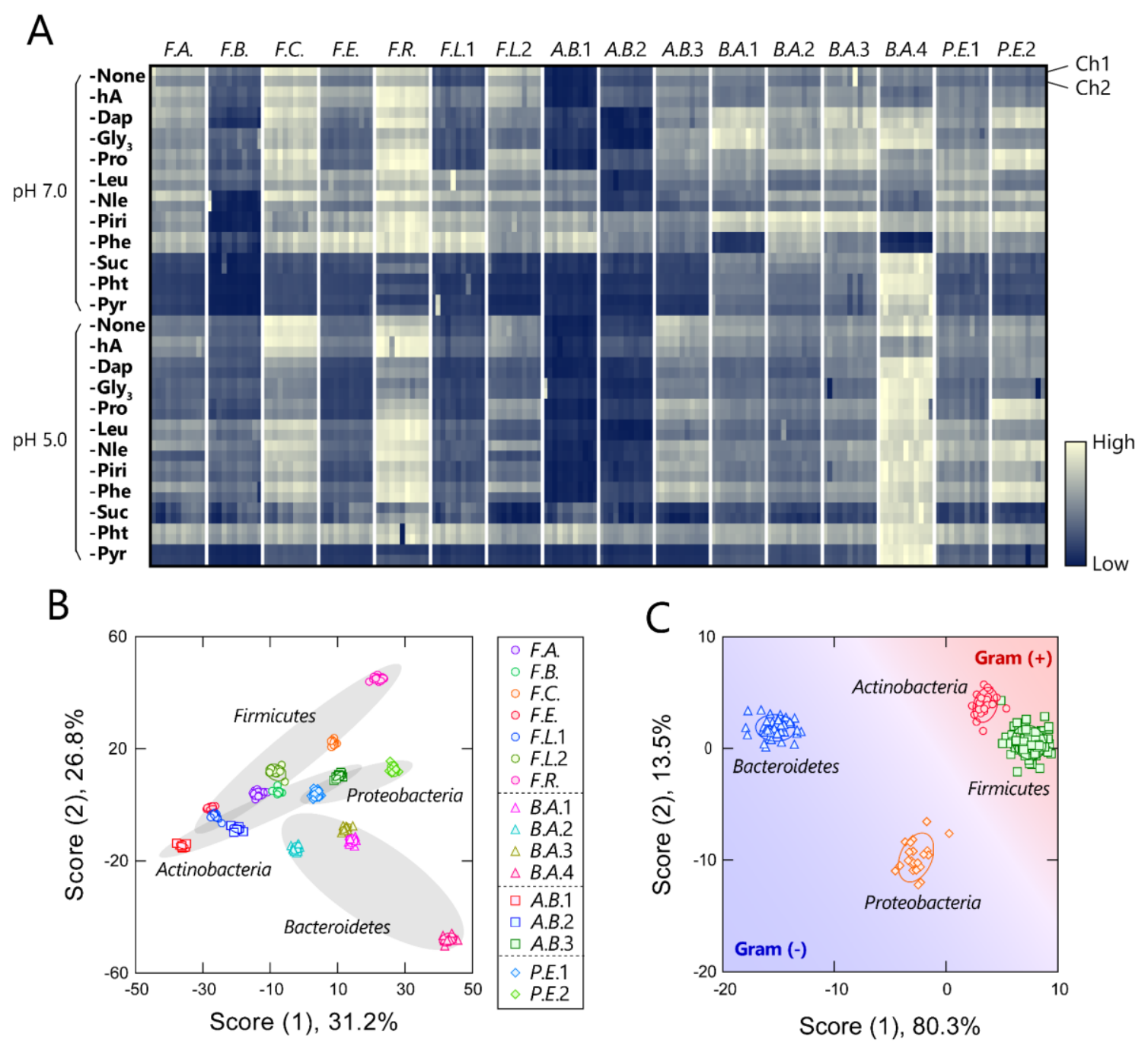

Figure 3. Optical pattern recognition of gut-derived bacteria. (A) Heat map of the fluorescence response patterns of 16 different intestinal bacterial strains $\left(\mathrm{OD}_{600}=0.04\right)$. Eleven replicates are shown for each analyte. $(B, C)$ Discriminant score plot for intestinal bacterial strains $\left(\mathrm{OD}_{600}=0.04\right)$, where analytes were labelled according to $(\mathrm{B})$ species and (C) phylum. Ellipsoids represent confidence intervals ( $\pm 1 \mathrm{SD})$ for each analyte. 
Intriguingly, clusters at the "phylum" level, which is a taxonomic rank much higher than the "species" level, were observed (Figure 3B), even though the bacterial phylotypes have not been well proven to correlate with their surface properties. To evaluate the potential of this phylumlevel feature extraction in more detail, a meta-analysis was performed with phylum labeling on the data for each bacterium. As expected, clusters corresponding to the four phyla segregated well on the linear discriminant score plot (Figure 3C). High accuracy was achieved in both the jackknife test $(99 \%)$ and the holdout test $(100 \%)$. Recent studies have been revealing that gut microbiota exhibit phylum- or genus-level compositional patterns ${ }^{10-15}$, suggesting that our chemical nose, which can recognize phylum-level features of intestinal bacterial strains, may serve to acquire the response patterns of gut microbiota. Note that our chemical tongue have succeeded in recognizing even differences between bacteria that are classified into the same "species" but differ at the lower "strains" level (i.e., eight Escherichia coli strains; Section 5 of the Supporting Information).

\section{Classification of models of obesity-associated gut microbiota}

Although still controversial, $F / B$ ratio in the gut microbiota is well known to vary between healthy humans/mice and those with lifestyle diseases, such as obesity and type II diabetes, and hence, gut microbiota composition even at the phylum level is expected to be a potential biomarker or a new therapeutic target for such diseases ${ }^{13-15}$. To investigate the applicability of our chemical nose to $F / B$ ratio classification, tests were performed using model gut microbiota that simulate obese and healthy samples. According to a recent report ${ }^{31}$, we prepared model gut microbiota consisting of six intestinal bacterial strains belonging to four major phyla, which differ in the content ratios of Firmicutes and Bacteroidetes (Figure 4A). These model microbiota were intended 
to cover the range from underweight persons $(F / B$ ratio $\sim 0.70$, nearly corresponding to "Slightlylow") to obese persons ( $F / B$ ratio $\sim 1.54$, nearly corresponding to "Slightly-high" $)^{31}$.

The prepared model microbiota were applied to a chemical nose composed of six polymers (-None, -Dap, -Gly3, -Leu, -Phe and -Pht), which were rationally chosen based on a hierarchical clustering analysis (for details, see Section 4 of the Supporting Information). Statistical analysis of the fluorescence response patterns (Figure S6) by LDA revealed that four clusters were distributed without overlapping in the two-dimensional space (Figure 4B). Consistently, the discriminant accuracies were $100 \%$ for the jackknife test and $97 \%$ for the holdout test. The first discriminant score [score (1)], which accounts for the most of variance (97.5\%), was highly correlated with $F / B$ ratio $(r=-0.95)$. This predictive behavior of the scores possibly leads to quantify $F / B$ ratios using regression analysis based on machine learning techniques ${ }^{16}$. Though this result is still preliminary because the real obesity-associated microbiota is far more complex, the ability to accurately recognize these subtle differences in bacterial mixtures may promise its application to real samples. 
A

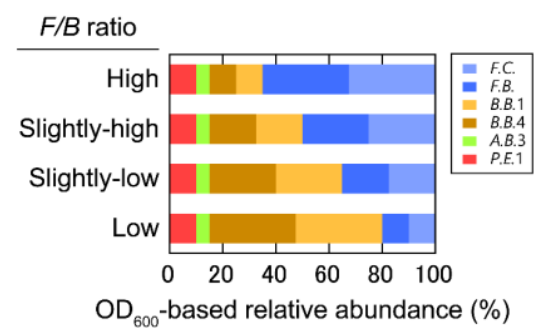

B

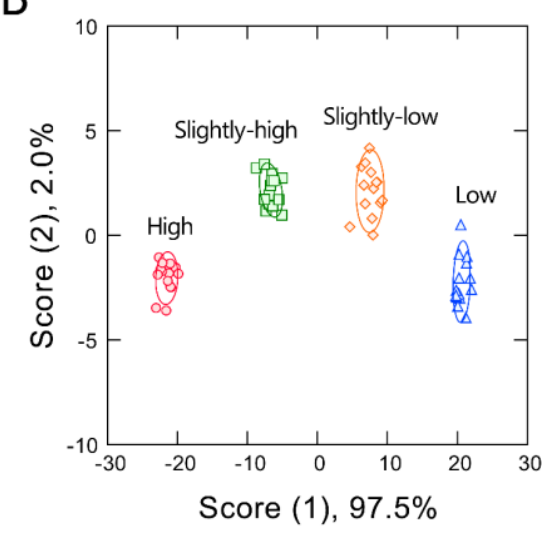

Figure 4. Optical pattern recognition of obese and healthy model bacterial mixtures.

(A) The relative abundance of intestinal bacterial strains in samples with different Firmicutes/Bacteroidetes ratios. (B) Discriminant score plot for intestinal bacterial mixtures, where ellipsoids represent confidence intervals $( \pm 1 \mathrm{SD})$ for each analyte.

\section{Application to the diagnosis of mouse insomnia}

One potential application of gut microbiome measurement is the diagnosis or routine monitoring of health status as well as that of serious disease development ${ }^{32,33}$. Here, we challenged a proof-of-concept study to detect deterioration in health conditions by optical pattern recognition of the microbiota in mouse feces. 
In this study, we employed a sleep disorder model to prepare mice with health deterioration, where continuous stress, disturbing sleep, is imposed on mice ( 8 weeks-aged) by being confined inside a rotating wheel ${ }^{34,35}$ for 28 days after a 10-day habituation process in a normal cage with the rotating wheel (Figures 5A and S7; for details, see Section 1 of the Supporting Information). In normal cages, nocturnal mice were significantly more active at night, whereas the stressed mice ran on the wheel almost all day immediately after exposure to the stress, causing sleep fragmentation (Figure 5B). Homogeneous microbiota suspensions were prepared as follows; fecal samples collected on day 28 from healthy or sleep-disordered mice ( $n=4$, respectively) were suspended in phosphate buffer saline (PBS), and then centrifuged to remove the soluble fraction, and filtered to remove large aggregates (for details, see Section 1 of the Supporting Information). The obtained gut microbiome samples ( 4 individuals $\times 2$ conditions) were diluted to $20 \mu \mathrm{g} / \mathrm{mL}$ by distilled water and used in subsequent experiments. 
A
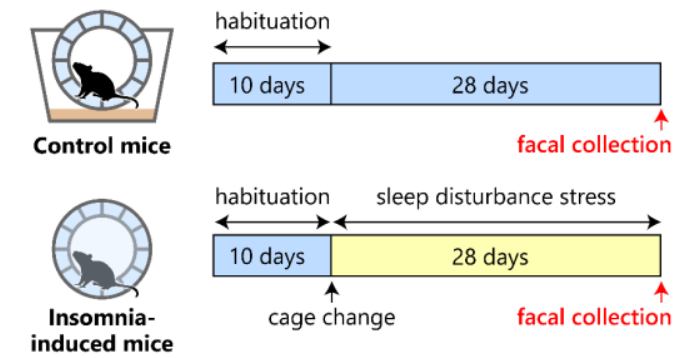

B

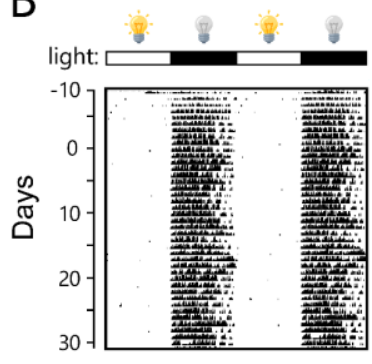

Control mice

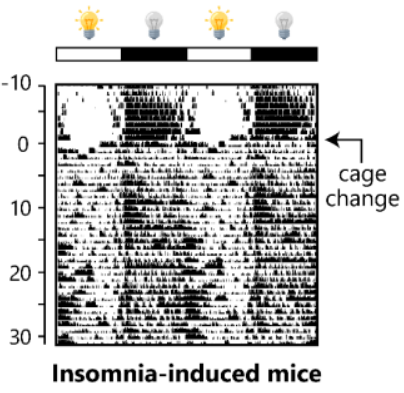

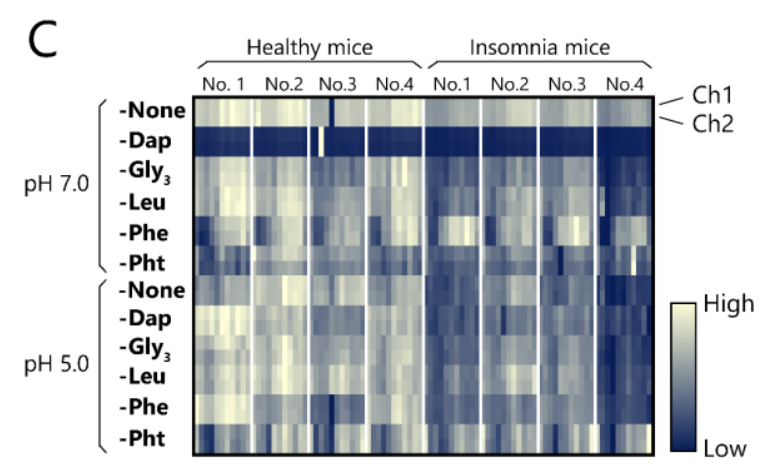

D

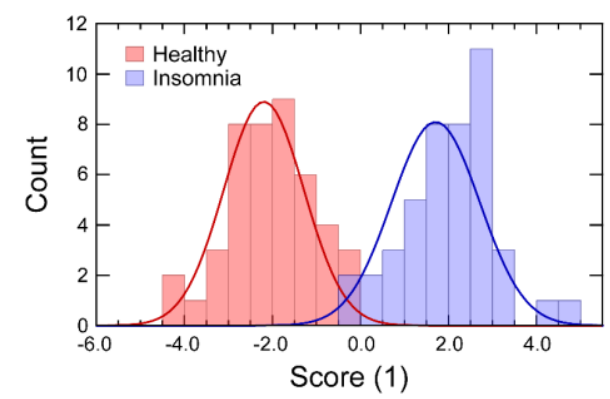

Figure 5. Optical pattern recognition of mouse gut microbiota. (A) Procedure of mouse fecal collection. After 10 days of habituation in the normal cage, mice for insomnia-induction were transferred to the sleep disturbance cage on day 0 , followed by the collection of mice feces after 28 days. (B) Representative double-plot actograms for control mice and those under sleep disturbance stress, which display in black while the mouse is rotating the wheel. The light/dark cycles are shown as white/black bars, respectively, above the actograms. (C) Heat map of the fluorescence response patterns of feces from healthy and insomnia mice (20 $\mu \mathrm{g} / \mathrm{mL}$ ). Eleven replicates are shown for each analyte. (D) Histogram of discriminant scores for mouse gut microbiota, which is marked with normal distributions fitted to the full data.

As with the axenic culture of gut-derived bacteria (Figure 2), addition of mouse gut microbiome samples to TPE-appended PEG- $b$-PLL markedly enhanced fluorescence emission (Figure S8). After confirming the response, we attempted to generate the fluorescence response 
patterns at 11 replicates for each sample by the chemical nose (Figure 5C). The LDA analysis of the thus-obtained twelve-dimensional patterns showed clear differences between the healthy and insomnia groups with only slight overlap on the histogram of discriminant scores (Figure 5D), where the Student's $t$-test indicated $p<1.8 \times 10^{-4}$ (Figure S9). Consistent with this differentiation, both the Jackknife test and the holdout test successfully afforded high reliability $(91 \%$ and $94 \%$, respectively). Interestingly, in an attempt to discriminate individual mice, little overlap was shown in the two-dimensional score plot (Figure S10) with 90\% accuracy for the jackknife test, even though these individuals grew up in the same rearing environment before the treatment. In this labeling, the clusters were again separated between healthy and insomnia mice, suggesting that the differences in responses between the presence/absence of the stress is larger than those between individuals. As well as the intestinal bacteria strains, we have successfully constructed a minimal sensor even for mouse gut microbiota (Section 4 of the Supporting Information).

\section{Discussion}

In the present study, we constructed a synthetic library of block copolymers appended with AIE fluorophores to establish a chemical nose strategy for sensing of gut microbiota. Our chemical nose composed of synthesized TPE-appended PEG-b-PLLs, which can produce marked fluorescence emission through binding to gut-derived bacteria, was capable of (i) identifying the species and the phyla of 16 intestinal bacterial strains (Figure 3) and the strains of eight Escherichia

coli strains (Figure S18), (ii) classifying artificial models (intestinal bacterial culture mixtures) of obesity-associated gut microbiota (Figure 4), and (iii) detecting sleep disorders through comparatively evaluating mouse gut microbiota (Figure 5). To the best of our knowledge, this 
study is the first in two respects; the chemical nose strategy allows (i) the recognition of phylum level differences and (ii) the applicability to the real complex gut microbiota (mouse fecal samples), which consist of more than 1,000 bacterial species ${ }^{1,2}$.

The achievement presented should be largely attributed to a newly developed polymer library that is suitable for microbiome-targeted chemical nose. TPE, which emits light in response to changes in molecular rotational motion, led to a high signal-to-background ratio ${ }^{24}$, offering high reproducibility and simplifying the sensing procedure. Twelve synthesized TPE-appended PEG$b$-PLLs cover a wide chemical space, which provides the ability to bind bacterial surfaces in a multi-contact manner that combines electrostatic, hydrophobic, $\pi-\pi$, and hydrogen-bonding interactions. This feature has more likely enabled to recognize patterns of characteristic microbiota composition correlated with the host conditions ${ }^{10-15}$. The 48 -dimensional optical response patterns generated by 12 polymers are one of the largest datasets in the hitherto chemical noses. Metaanalysis of these dataset will provide more in-depth knowledge and guide new material designs for chemical noses.

To date, the chemical nose strategy has been applied to detect pathogenic bacteria in the context of diagnosing infectious diseases ${ }^{36}$, where libraries of fluorescent probe molecules ${ }^{26,27,37}$, polymers $^{38}$, nanoparticles ${ }^{39,40}$ and supramolecular complexes ${ }^{41,42}$ have been developed to identify the pathogens. However, no chemical nose approach for gut-derived bacteria has been developed and reported yet. Besides, our system can discriminate not only the species of these bacteria, but also the phylum level, a much higher phylogenetic rank (Figure 4C). The outer surface of grampositive bacteria [Gram (+); Actinobacteria and Firmicutes] is composed of peptidoglycans that are typically intercalated with covalently attached anionic teichoic acids, while that of gramnegative bacteria [Gram (-); Bacteroidetes and Proteobacteria] is surrounded by a negatively- 
charged outer membrane containing lipopolysaccharides ${ }^{25}$. These outer structures produce large physicochemical differences, which should have been recognized by our chemical nose, similar to the previous reports $37,41,42$. However, the common differences in the outer structures between bacteria bearing the same gram-stainability are not well understood. Therefore, the ability to extract information about such unknown differences is a distinguishing feature of our system.

Our current chemical nose is capable of measuring relatively small amount of samples $(<10$ $\mu \mathrm{g})$ in a short time $(<30 \mathrm{~min})$ with only a common analytical instrument, and comparative analysis can be performed based on the overall characteristics of the samples even if the details of the target microbiota are unknown. Since this system does not need to grow bacteria and can directly measure the real environmental samples, it will be promising for application to a variety of bacteria and microbiomes, including fastidious and/or uncultured bacteria, which are a major obstacle to microbiology and its-related industries. Therefore, we expect our approach to complement the metagenomic analysis to offer a powerful platform for simple, accurate, and high-throughput characterization of microbiota in a wide range of fields, including medical and healthcare.

\section{Corresponding Authors}

*E-mail: s.tomita@aist.go.jp (S.T.).

*E-mail: r.kurita@aist.go.jp (R.K.).

\section{Acknowledgements}


We thank Dr. Masahiko Nakamoto (Department of Chemistry, University of California, Irvine) for help with acid-base titration experiments. This work was partially supported by JSPS KAKENHI grant (JP17H04884 and JP20H02774 to S.T.; JP19H05679 and JP19H05683 (PostKoch Ecology) to H.K. and H.T.), AMED PRIME (Grant number JP18gm6010019) to H.T., JST ERATO Grant Number JPMJER1502 to H.T., and DAICENTER project grant from the DBT (Govt. of India) to Renu Wadhwa and special strategic grant from AIST (Japan) to R.K.

\section{References}

1. Qin, J. et al. A human gut microbial gene catalogue established by metagenomic sequencing. Nature 464, 59-65 (2010).

2. Almeida, A. et al. A new genomic blueprint of the human gut microbiota. Nature 568, 499504 (2019).

3. Lynch, S. V. \& Pedersen, O. The human intestinal microbiome in health and disease. $N$. Engl. J. Med. 375, 2369-2379 (2016).

4. Rothschild, D. et al. Environment dominates over host genetics in shaping human gut microbiota. Nature 555, 210-215 (2018).

5. Poroyko, V. A. et al. Chronic sleep disruption alters gut microbiota, induces systemic and adipose tissue inflammation and insulin resistance in mice. Sci. Rep. 6, 35405 (2016).

6. Riquelme, E. et al. Tumor microbiome diversity and composition influence pancreatic cancer outcomes. Cell 178, 795-806 (2019).

7. Helmink, B. A., Khan, M. A. W., Hermann, A., Gopalakrishnan, V. \& Wargo, J. A. The microbiome, cancer, and cancer therapy. Nat. Med. 25, 377-388 (2019).

8. Nayfach, S. \& Pollard, K. S. Toward accurate and quantitative comparative metagenomics. Cell 166, 1103-1116 (2016).

9. Fricker, A. M., Podlesny, D. \& Fricke, W. F. What is new and relevant for sequencing-based 
microbiome research? A mini-review. J. Adv. Res. 19, 105-112 (2019).

10. Arumugam, M. et al. Enterotypes of the human gut microbiome. Nature 473, 174-180 (2011).

11. Costea, P. I. et al. Enterotypes in the landscape of gut microbial community composition. Nat. Microbiol. 3, 8-16 (2018).

12. Christensen, L., Roager, H. M., Astrup, A. \& Hjorth, M. F. Microbial enterotypes in personalized nutrition and obesity management. Am. J. Clin. Nutr. 108, 645-651 (2018).

13. Turnbaugh, P. J. et al. An obesity-associated gut microbiome with increased capacity for energy harvest. Nature 444, 1027-1031 (2006).

14. Sweeney, T. E. \& Morton, J. M. The human gut microbiome: A review of the effect of obesity and surgically induced weight loss. JAMA Surg. 148, 563-569 (2013).

15. Abenavoli, L. et al. Gut microbiota and obesity: A role for probiotics. Nutrients 11, 2690 (2019).

16. Li, Z., Askim, J. R. \& Suslick, K. S. The optoelectronic nose: Colorimetric and fluorometric sensor arrays Chem. Rev. 119, 231-292 (2019).

17. Sugai, H., Tomita, S. \& Kurita, R. Pattern-recognition-based sensor arrays for cell characterization: From materials and data analyses to biomedical applications. Anal. Sci. https://doi.org/10.2116/analsci.20R002 (2020).

18. Tomita, S., Sakao, M., Kurita, R., Niwa, O. \& Yoshimoto, K. A polyion complex sensor array for markerless and noninvasive identification of differentiated mesenchymal stem cells from human adipose tissue. Chem. Sci. 6, 5831-5836 (2015).

19. Tomita, S., Niwa, O. \& Kurita, R. Artificial modification of an enzyme for construction of cross-reactive polyion complexes to fingerprint signatures of proteins and mammalian cells. Anal. Chem. 88, 9079-9086 (2016).

20. Tomita, S. et al. Noninvasive fingerprinting-based tracking of replicative cellular senescence using a colorimetric polyion complex array. Anal. Chem. 90, 6348-6352 (2018).

21. Rana, S. et al. Array-based sensing of metastatic cells and tissues using nanoparticlefluorescent protein conjugates. ACS Nano. 6, 8233-8240 (2012). 
22. Le, N. D. B. et al. Cancer cell discrimination using host-guest "doubled" arrays. J. Am. Chem. Soc. 139, 8008-8012 (2017).

23. Tomita, S., Ishihara, S. \& Kurita, R. Biomimicry recognition of proteins and cells using a small array of block copolymers appended with amino acids and fluorophores. ACS Appl. Mater. Interfaces 11, 6751-6758 (2019).

24. Mei, J., Leung, N. L., Kwok, R. T., Lam, J. W. \& Tang, B. Z. Aggregation-induced emission: Together we shine, united we soar! Chem. Rev. 115, 11718-11940 (2015).

25. Silhavy, T. J., Kahne, D. \& Walker, S. The bacterial cell envelope. Cold Spring Harb. Perspect. Biol. 2, a000414 (2010).

26. Liu, G.J., Tian, S. N., Li, C. Y., Xing, G. W. \& Zhou, L. Aggregation-induced-emission materials with different electric charges as an artificial tongue: Design, construction, and assembly with various pathogenic bacteria for effective bacterial imaging and discrimination. ACS Appl. Mater. Interfaces 9, 28331-28338 (2017).

27. Zhou, C. et al. Engineering sensor arrays using aggregation-induced emission luminogens for pathogen identification Adv. Funct. Mater. 1805986 (2018).

28. Tomita, S., Ishihara, S. \& Kurita, R. Environment-sensitive turn-on fluorescent polyamino acid: Fingerprinting protein populations with post-translational modifications. ACS Appl. Mater. Interfaces 9, 22970-22976 (2017).

29. Rosenbaum, M., Knight, R. \& Leibel, R. L. The gut microbiota in human energy homeostasis and obesity. Trends Endocrinol. Metab. 26, 493-501 (2015).

30. Anzenbacher Jr, P., Lubal, P., Bucek, P., Palacios, M. A. \& Kozelkova, M. E. A practical approach to optical cross-reactive sensor arrays. Chem. Soc. Rev. 39, 3954-3979 (2010).

31. Koliada, A. et al. Association between body mass index and Firmicutes/Bacteroidetes ratio in an adult Ukrainian population. BMC Microbiol. 17, 120 (2017).

32. Lloyd-Price, J., Abu-Ali, G. \& Huttenhower, C. The healthy human microbiome. Genome Med. 8, 51 (2016).

33. Johnson, A. J. et al. Daily sampling reveals personalized diet-microbiome associations in humans. Cell Host Microbe 25, 789-802 (2019). 
34. Miyazaki, K., Itoh, N., Ohyama, S., Kadota, K. \& Oishi, K. Continuous exposure to a novel stressor based on water aversion induces abnormal circadian locomotor rhythms and sleepwake cycles in mice. PLoS One 8, e55452 (2013).

35. Minakawa, E. N. et al. Chronic sleep fragmentation exacerbates amyloid beta deposition in Alzheimer's disease model mice. Neurosci. Lett. 653, 362-369 (2017).

36. Chen, J., Andler, S. M., Goddard, J. M., Nugen, S. R. \& Rotello, V. M. Integrating recognition elements with nanomaterials for bacteria sensing. Chem. Soc. Rev. 46, 12721283 (2017).

37. Svechkarev, D., Sadykov, M. R., Bayles, K. W. \& Mohs, A. M. Ratiometric fluorescent sensor array as a versatile tool for bacterial pathogen identification and analysis. ACS Sens. 3, 700-708 (2018).

38. Ngernpimai, S. et al. Rapid identification of biofilms using a robust multichannel polymer sensor array. ACS Appl. Mater. Interfaces 11, 11202-11208 (2019).

39. Li, B. et al. Colorimetric sensor array based on gold nanoparticles with diverse surface charges for microorganisms identification. Anal. Chem. 89, 10639-10643 (2017).

40. Ji, H., Wu, L., Pu, F., Ren, J. \& Qu, X. Point-of-care identification of bacteria using proteinencapsulated gold nanoclusters. Adv. Healthc. Mater. 7, e1701370 (2018).

41. Han, J. et al. A polymer/peptide complex-based sensor array that discriminates bacteria in urine. Angew. Chem. Int. Ed. 56, 15246-15251 (2017).

42. Shen, J. et al. Fluorescent sensor array for highly efficient microbial lysate identification through competitive interactions. ACS Sens. 3, 2218-2222 (2018).

43. Thompson, C. C. et al. Microbial taxonomy in the post-genomic era: Rebuilding from scratch? Arch. Microbiol. 197, 359-370 (2015).

44. Hayashi Sant'Anna, F. et al. Genomic metrics made easy: what to do and where to go in the new era of bacterial taxonomy. Crit. Rev. Microbiol. 45, 182-200 (2019).

45. The Human Microbiome Project, Consortium structure, function and diversity of the healthy human microbiome. Nature 486, 207-214 (2012).

46. Jansson, J. K. \& Hofmockel, K. S. Soil microbiomes and climate change. Nat. Rev. 
Microbiol. 18, 35-46 (2020).

47. Busby, P. E. et al. Research priorities for harnessing plant microbiomes in sustainable agriculture. PLoS Biol. 15, e2001793 (2017).

48. Fierer, N. Embracing the unknown: disentangling the complexities of the soil microbiome. Nat. Rev. Microbiol. 15, 579-590 (2017).

49. Wang, L. \& Li, X. Steering soil microbiome to enhance soil system resilience. Crit. Rev. Microbiol. 45, 743-753 (2019).

50. Qiu, Z., Egidi, E., Liu, H., Kaur, S. \& Singh, B. K. New frontiers in agriculture productivity: Optimised microbial inoculants and in situ microbiome engineering. Biotechnol. Adv. 37, 107371 (2019). 